\title{
A Non-Linear Flash Analog to Digital Architecture for Sinusoidal Input Signals
}

\author{
Charles A. Berdanier \\ AFRL/RYRT
}

\author{
John Scanlan \\ AFRL/RYRT
}

\begin{abstract}
We hypothesize that we can improve the footprint by using a static non-linear flash encoding scheme for Analog to Digital $(A / D)$ converters for an important set of functions. This architecture can reduce the number of comparators and resisters in a flash converter by $25 \%$.
\end{abstract}

Keywords: Flash, Analog to Digital, Conversion, Mixed signals

\section{Introduction}

Traditional Flash A/D converters have historically been among the fastest and simplest $\mathrm{A} / \mathrm{D}$ architectures but use in recent years has been limited by power, heat and resolution limits resulting from the large number of components needed for implementation. This paper will present one method for reducing the component count for flash $\mathrm{A} / \mathrm{D}$ converters for a set of signals.

\section{Reduced Comparator Flash A/Ds}

Flash converters are extremely fast compared to other types of $\mathrm{A} / \mathrm{D}$ converters but have traditionally required a huge number of comparators compared to other $\mathrm{A} / \mathrm{D}$ converter architectures. In a traditional flash A/D the architecture consists of linear voltage ladder with a comparator at each voltage of the voltage divider circuit. These comparators are directly connected to the encoder logic which allows some engineers to refer to flash $A / D s$ as direct conversion because the conversion is done in one stage without iteration.[1] [2]

Flash conversion is simple but also is resource intensive requiring $2^{\mathrm{a}}-1$ comparators and resistors in the first stage (where a is the effective number of bits for the converter) This has effectively limited the construction of flash A/Ds to eight bits or less due to heat, power and density constraints. [3]

\subsection{Previous work}

Previous investigators into non linear encoding techniques have heavily concentrated on low frequency, voice transmission with an emphasis on the compression and decompression of voice signals for telephone

U.S. Government work not protected by U.S. copyright transmission. [4] [5]. The American standard for compressive A/D, $\mu$ Law, is able to compress a 13 bit resolution into an 8 bit encoding with no audible distortion. This is due to the sensitivity of human hearing rather than the accuracy of the conversion.

Non-linear A/Ds have also been used in low frequency Sonar applications where resolution needs to be as high as possible but conversion rate is not an issue. [6][7]

\subsection{Linear Flash A/D Architectures}

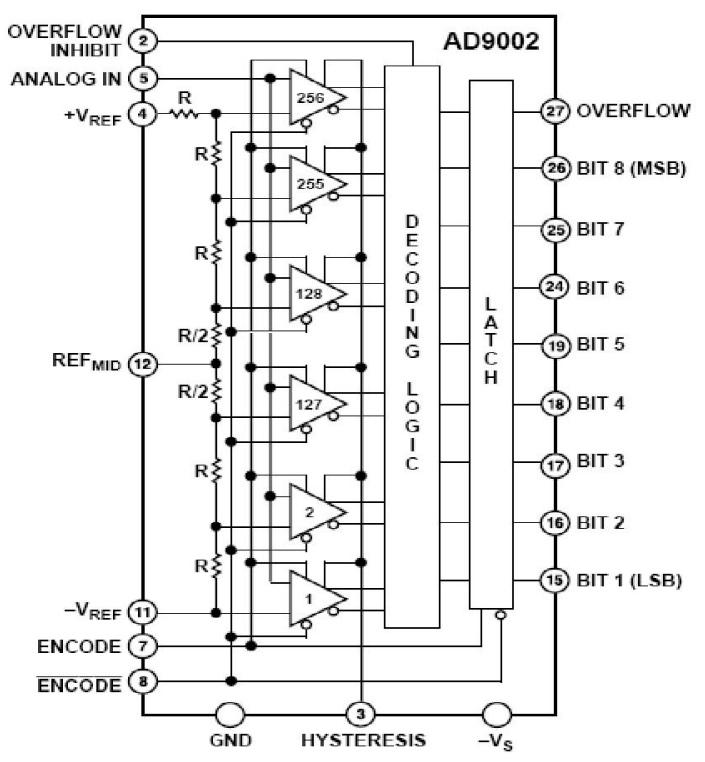

Figure 1 Analog Devices AD9002 a typical 8 bit flash converter architecture [8]

The Analog Devices AD9002 flash A/D converter, shown above, is typical of the class. It has a typical voltage range of 2 volts and eight bit resolution. The voltage divider network, visible at the left of the figure, is linear and divides the two into 256 steps of $7.8125 \mathrm{mV}$

\subsection{High Speed Non-Linear A/Ds Signals}

Since most prior non linear A/D work has been heavily concentrated on telephony, there is not much written 
about other non-linear A/D applications. The requirement is to find a distribution of signals that are non-uniform across the voltage range and matching the resolution to the frequency of the occurrence. This condition can be satisfied by a simple sine wave function that covers the full range of the A/D converter. If we divide a $2 \mathrm{~V}$ input range of an $\mathrm{A} / \mathrm{D}$ into $500 \mathrm{mV}$ quadrants and examine a sine wave input (see figure 2) we see the bulk of the samples are found in the top and bottom quadrants.

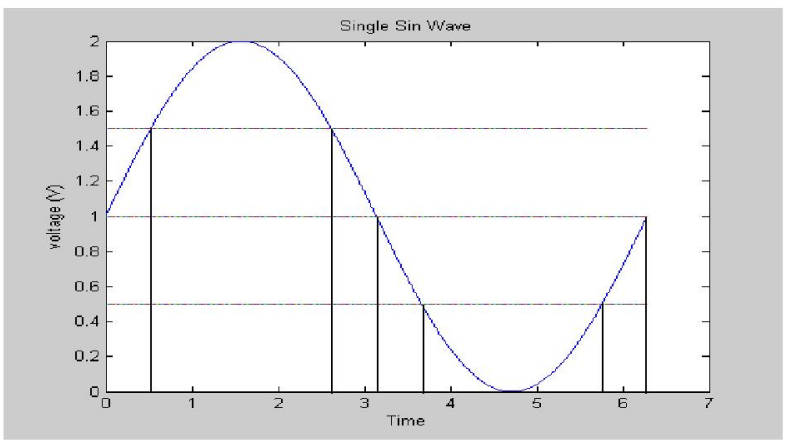

Figure 2 A full range sine wave

The values from figure 1 shows the samples from zero to $/ 6$ and from $5 / 6$ to in the voltage range between $1 \mathrm{~V}$ and $1.5 \mathrm{~V}$ this compares to the other $2 / 3 \mathrm{~s}$ of in the range from $1.5 \mathrm{~V}$ and $2 \mathrm{~V}$. There is a similar amount of time form the zones between $0.5 \mathrm{~V}$ and $1 \mathrm{~V}$ and the voltage zone between $0 \mathrm{~V}$ and $0.5 \mathrm{~V}$. As histogram of these sample is shown in figure 2.

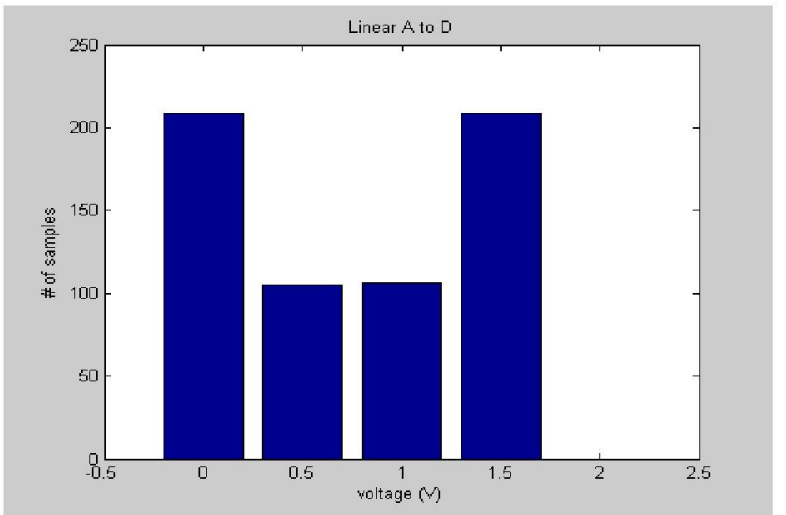

Figure $3 \mathrm{~A}$ histogram by voltage range of fig 2

\subsection{A Non-linear Flash A/D}

Because a full scale sinusoidal signal (or any signal with a sinusoidal mode) will spend two thirds of it's cycle in the top and bottom voltage zones we can propose a new nonlinear architecture to take advantage of that fact. If we use 64 comparators from $2.0 \mathrm{~V}$ to $1.5 \mathrm{~V}$ and also from $0.5 \mathrm{~V}$ to $0.0 \mathrm{~V}$ we will have the same number of comparators as a normal eight bit flash converter in those voltage ranges. If we decrease the number of comparators in the middle range, $1.5 \mathrm{~V}$ to $0.5 \mathrm{~V}$, from 128 comparators to 64 comparators we will be reducing the total number of comparators in the design from 256 to 192 . The data will still be in an eight bit output format with a slightly increased quantization error over the entire voltage range. Importantly, we will only be affecting one third of all samples for full range sinusoidal signals and the total effect will be to double the quantization error for half of those samples.

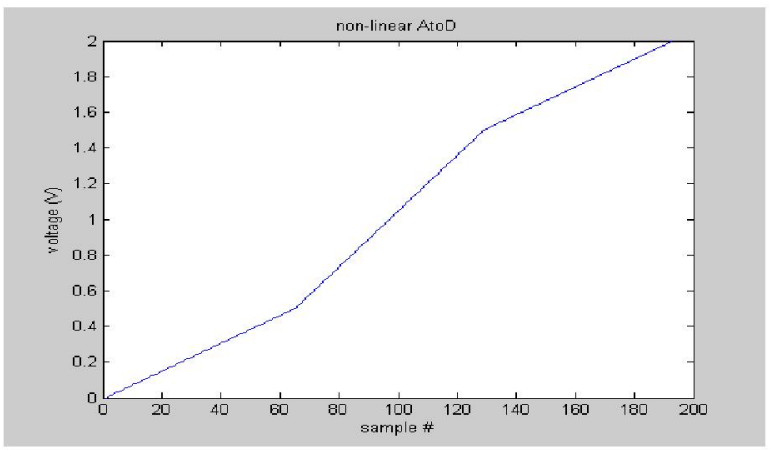

Figure 4 Non-Linear voltage transfer

When we digitize the sine wave into 200 samples using this encoding scheme and the linear then compare the differences between the linear and non-linear $A / D$ we see an average of 2 samples per non-linear bin difference in the $0.5 \mathrm{~V}$ to $1.5 \mathrm{~V}$ voltage range. These 33 samples have twice the quantization error of the other samples due to the lack of resolution in the least significant bit but only represent $18.9 \%$ of all the samples. In these cases the quantization error is $7.8125 \mathrm{mV}$. In the 415 other samples the quantization error is $3.9062 \mathrm{mV}$ for an average quantization error of $4.6463 \mathrm{mV}$.

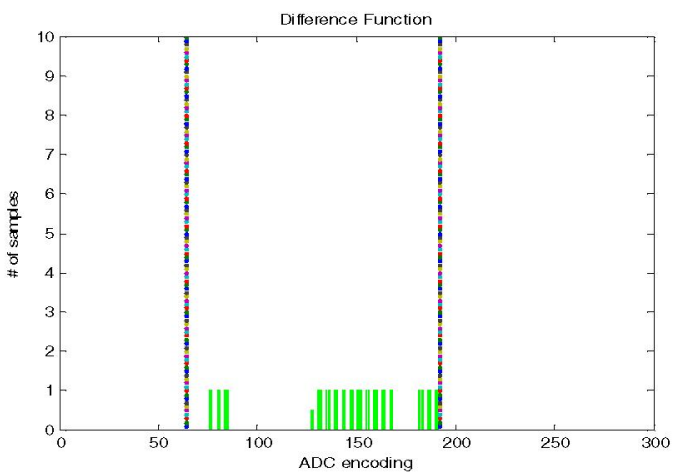

Figure 5 Linear / Non-linear encoding differences

If we compare the plot of the actual versus the linear and the nonlinear encoding over five cycles of the sine wave we get the following plot. 


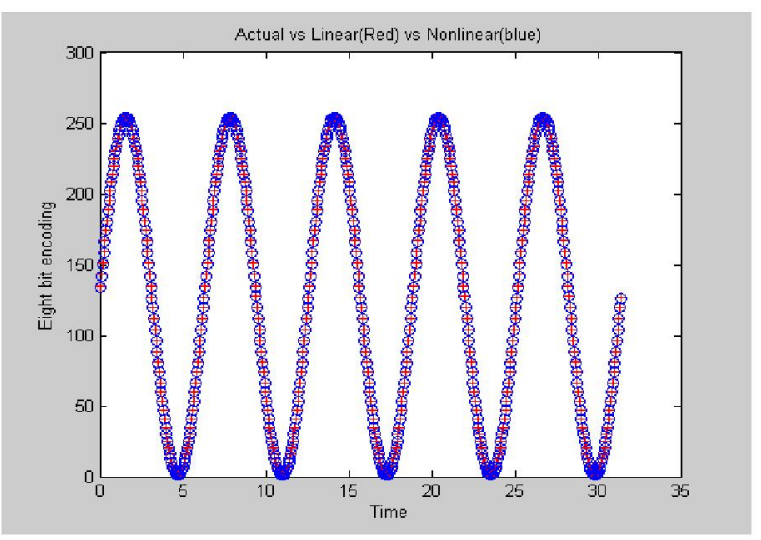

Figure 6 Encoding of the sine wave by a linear vs. a non linear $\mathrm{ADC}$

The majority of the errors will be found in the range from encoding 64 to 192 ands if we zoom into that area we can see small quantization errors consistent with the theoretical case described earlier.

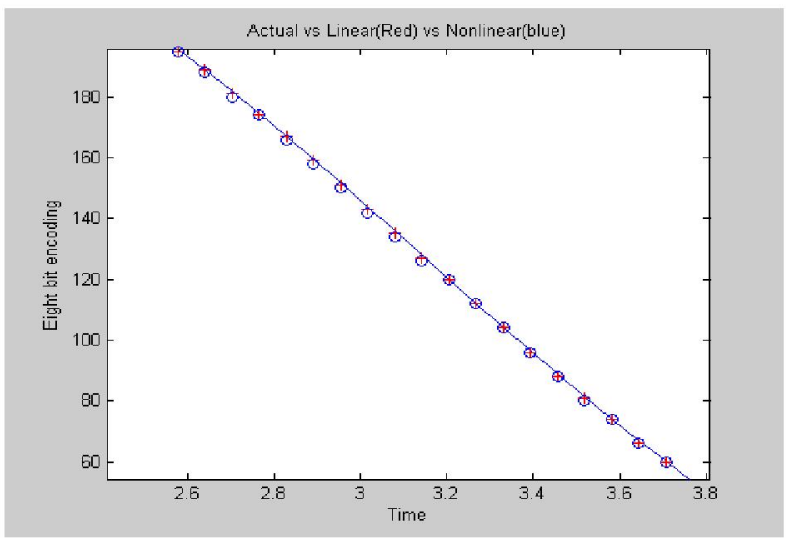

Figure 7 A close up view of the quantization errors of both the Linear and Nonlinear encoding

\subsection{A Two Frequency Example}

A practical application of this non-linear approach can be found in the detection of a $-10 \mathrm{~dB}$ signal $3 \mathrm{kHz}$ away from a full scale $100 \mathrm{kHz}$ sin wave. From inspection we can see that the signal meets the criteria listed earlier.

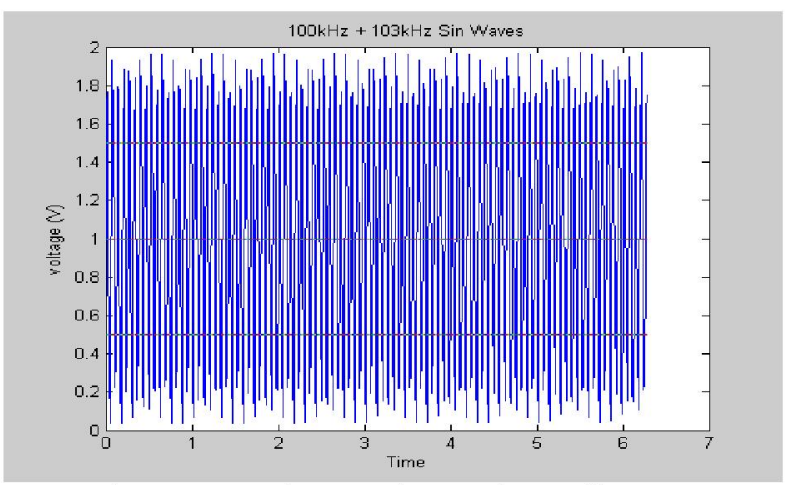

Figure 8 Two frequencies $-10 \mathrm{~dB}$ smaller one

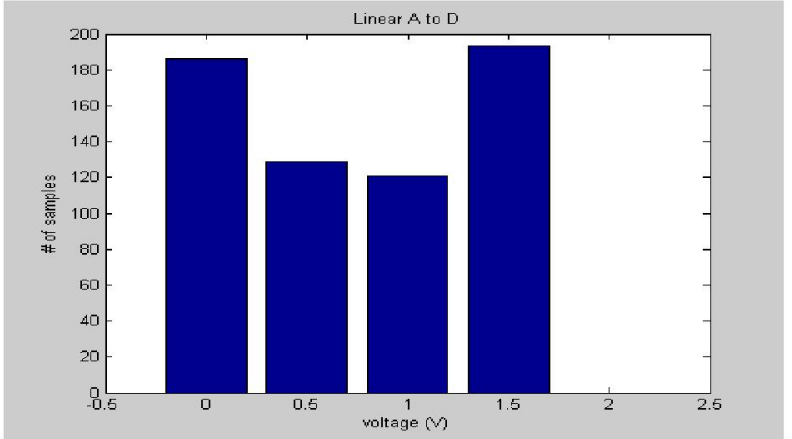

Figure 9 Histogram of the two frequency signal

By inspection the amplitude of the combined signal is nearly full scale without clipping. It is sinusoidal by design and the four part histogram shows a significant distribution of samples in the two outer voltage ranges. If we examine the histogram of the linear encoding of the signal we see an expected distribution.

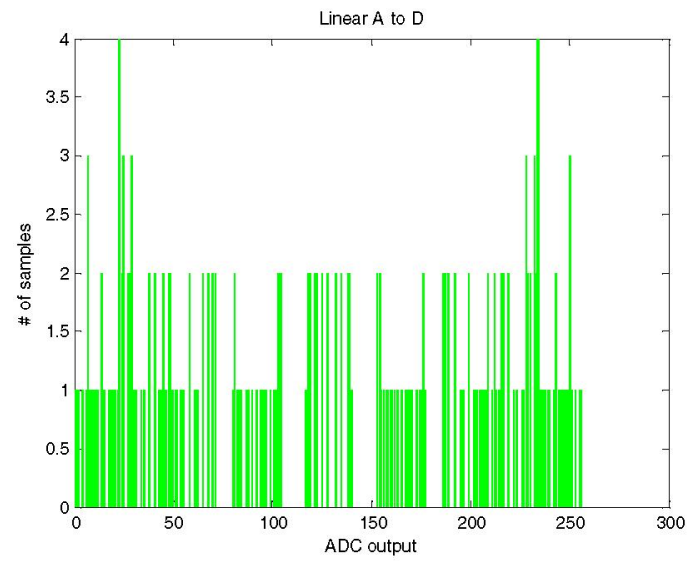

Figure 10 Histogram of the linear encoding of the signal

The nonlinear case also produces a similar histogram

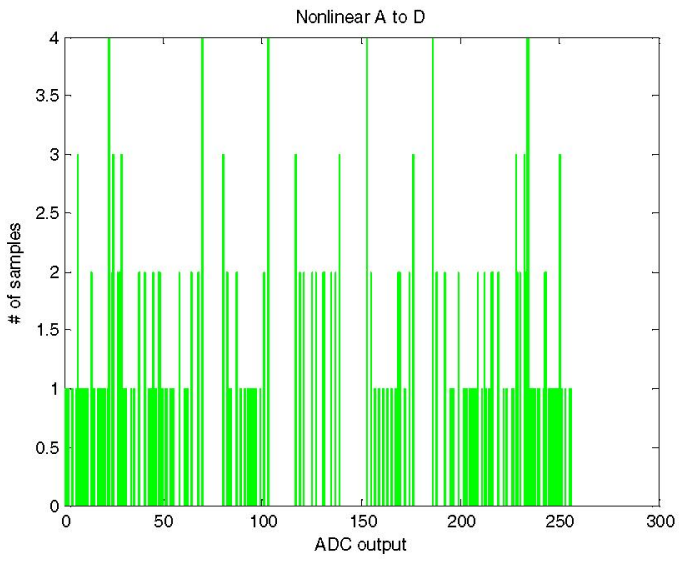


Figure 11 Histogram of the nonlinear encoding of the signal

The difference function for these two signals is similar to the single sine wave function.

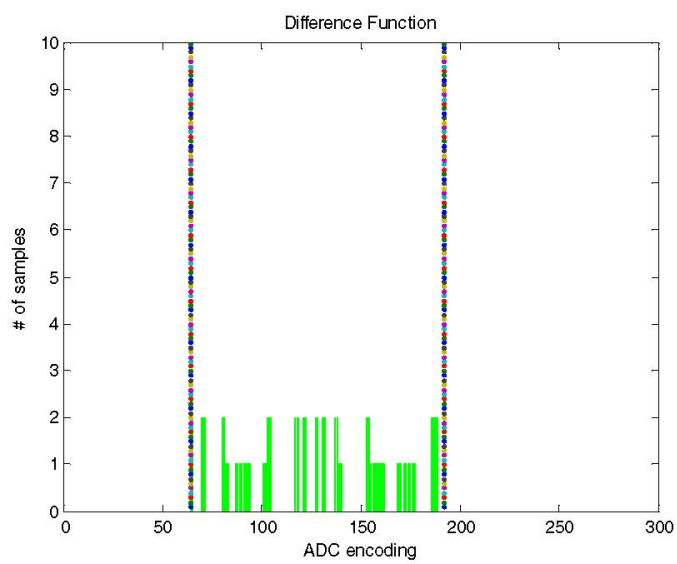

Figure 12 Difference function for the two frequency case

When the signal is reconstructed the errors are limited to the least significant bit.

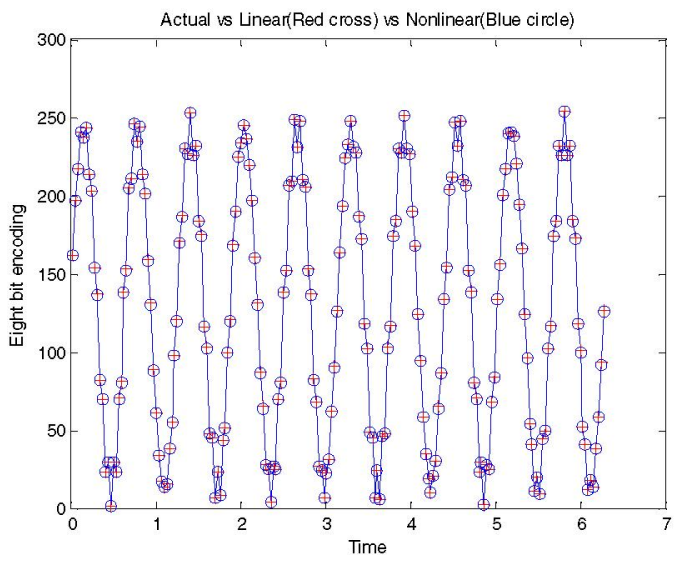

\section{Future Directions}

Further validation of this idea could be accomplished via a breadboard experiment. Many ADC evaluation boards are equipped with additional output pins that allow for signal evaluation and augmentation. Many of the tests presented in this paper could be programmed into an FPGA card as interface for control and analysis. Furthermore, ADC behavioral modeling software is often available from vendors such as Analog Devices, further defining the data converter architecture, and how novel converter circuit designs could be implemented. [9]

\section{Conclusions}

For a limited set of functions, this reduced flash converter may provide near the resolution of an eight bit linear converter but uses only $75 \%$ of the resources. Both the architecture and the signal types it's suitable for remain subjects for further research. The authors of this paper would like to acknowledge Dr. Charles Cerny and Mr. Tom Niedzwiecki for their constructive insights during technical discussions that have shaped this topic area. This effort was funded through an Air Force Research Laboratory Entrepreneurial research award under in-house task $2002 \mathrm{IHOH}$.

\section{References}

[1] Digital Logic:Analysis, Applications and Design, Garrod and Borns, Saunders College Publishing, pg 928

[2] Electrical Engineering Handbook, Dorf, CRC Press, pp 777-778

[3] AN-215A Designer's Guide to Flash-ADC Testing, Kester, Analog Devices, 1990

[4] Communications Systems, Haykin, Wiley, 1983, pg 411

[5] Ibid, pg 412

[6] ICASSP, IEEE International Conference on Acoustics, Speech and Signal Processing - Proceedings, v 3, 2007 IEEE International Conference on Acoustics, Speech and Signal Processing, ICASSP '07, p III 1433 III 1436

[7] Externally linear, time-invariant systems and their application to companding signal processors, Tsividis, Y., Circuits and Systems II: Analog and Digital Signal Processing, IEEE Transactions on, Vol. 44, no. 2, pp.6585, Feb 1997

[8] AD9002 High Speed 8-Bit Monolithic A/D Converter Datasheet, Analog Devices, 2003

[9] AD9446 16-Bit, 80/100MSPS, A/D Converter Preliminary Datasheet, Analog Devices, 2005 